\title{
Acute marginal branch occlusion presenting with anterior wall ST segment elevation during percutaneous coronary intervention of the mid right coronary artery
}

\section{Kent A Tan*and Chan K Hui \\ Department of Cardiology, National University Heart Centre, Singapore}

\section{Case}

A 78-year-old male, ex-smoker, with a history of hypertension and hyperlipidemia presented with a one-month duration of worsening angina on exertion. Standard 12 L ECG showed sinus rhythm with early repolarization pattern in the anterolateral leads. Coronary angiography showed minor luminal irregularities in the left coronary vessels (Figure 1 A \& B) while the RCA showed high grade non-thrombotic $90 \%$ lesion in its mid segment (Figure 2A arrow) followed by another high grade $90 \%$ stenosis in the RPDA branch (Figure 2B). A small calibre acute marginal branch (AMB) with an $80 \%$ ostial stenosis was noted to take off from the diseased mid RCA segment (Figure 2A arrowhead). Ad hoc percutaneous coronary intervention (PCI) of both the RPDA and mid RCA was carried out.

After predilation, a $2.5 \times 24 \mathrm{~mm}$ SYNERGY (Boston Scientific) Everolimus drug eluting stent was implanted at the RPDA stenosis followed by another $3.5 \times 24 \mathrm{~mm}$ SYNERGY (Boston Scientific) Everolimus drug eluting stent at the mid RCA lesion. Following stent postdilation, repeat angiography demonstrated loss of the AMB from the mid RCA. (Figure 3). The patient subsequently developed chest pain and 12L ECG showed ST elevation in leads V1-V4 from baseline, most marked in V2 and V3. (Figure 4)

Attempts to salvage the jailed AMB were unsuccessful due to its angulated origin. IV analgesia with midazolam and fentanyl alleviated the chest pain. The patient remained hemodynamically stable, hence decision was made to stop the procedure and continue optimal medical therapy. At the coronary care unit, bedside transthoracic echocardiogram revealed no anterior wall motion abnormality to suggest LAD occlusion. There was significant increase in serum Troponin I level to $9091 \mathrm{ng} / \mathrm{mL}$ after 24 hours. Serial 12L ECG monitoring however showed gradual decline of ST elevation in leads V1- V4 (Figures 5 A \& B). Resolution of STE was noted nine (9) hours post-procedure (Figure 5C). Repeat transthoracic echocardiogram the following day showed normal LV and RV function with an ejection fraction of $60 \%$ with no segmental wall motion abnormalities. Medical therapy was further optimized, and patient was discharged well 3 days after PCI.

\section{Discussion}

Our case demonstrated onset of anterior leads (V1-V4) STE with no changes in the limb leads tracing after AMB loss following mid RCA stent implantation. The existing high- grade ostial AMB stenosis predisposed to its closure which resulted in isolated RV infarction (IRVI). RV myocardial infarction most often occurs simultaneously with an inferior LV wall infarction involving an occlusion of the

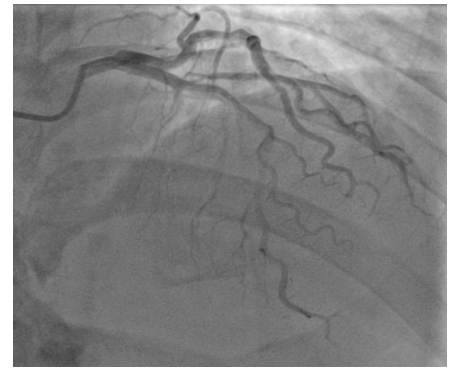

A

Figure 1. A. LAD and B. Non-dominant LCx, both vessels with minor irregularities
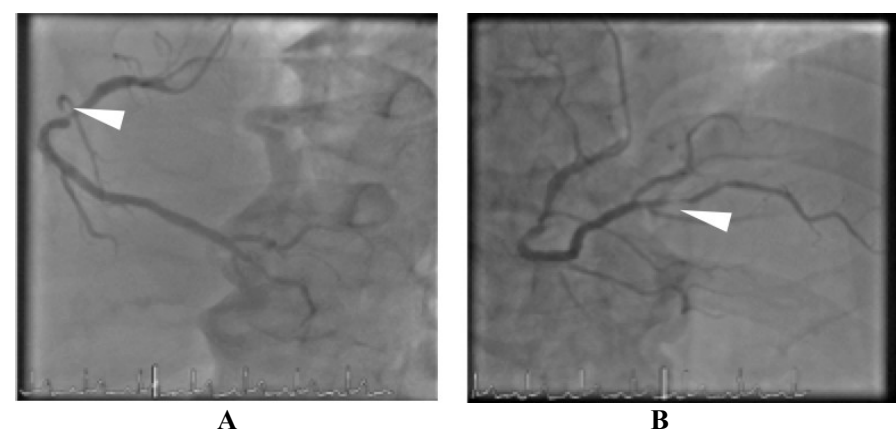

Figure 2. A. Mid RCA stenosis (arrow); Small caliber AMB taking off from diseased mid RCA segment (arrowheads). B. RPDA stenosis (arrow). AMB (acute marginal branch), RCA (right coronary artery), RPDA (right posterior descending artery)

proximal dominant RCA [1]. Left precordial ECG manifestations of RV injury are often absent because they are masked by dominant forces of the accompanying LV inferior wall injury $[2,3]$. Hence, the predominant ECG presentation of ST elevation (STE) in the inferior limb leads. STE in V4R identifies patients with an acute inferior wall myocardial infarction who are likely to have concomitant RV involvement [1].

However, a larger part of the right ventricle free wall is directed anteriorly and when myocardial injury is isolated only to the right

${ }^{\star}$ Correspondence to: Kent Anthony Tan, Interventional Cardiology, Department of Cardiology, National University Heart Centre, 1E Kent Ridge Road, NUHS Tower Block, Level 9, 119228, Singapore, Tel: (65) 6772 5211, E-mail: anthony_ tan@nuhs.edu.sg

Received: September 18, 2020; Accepted: September 29, 2020; Published: October 08, 2020 
Tan KA (2021) Acute marginal branch occlusion presenting with anterior wall ST segment elevation during percutaneous coronary intervention of the mid right coronary artery

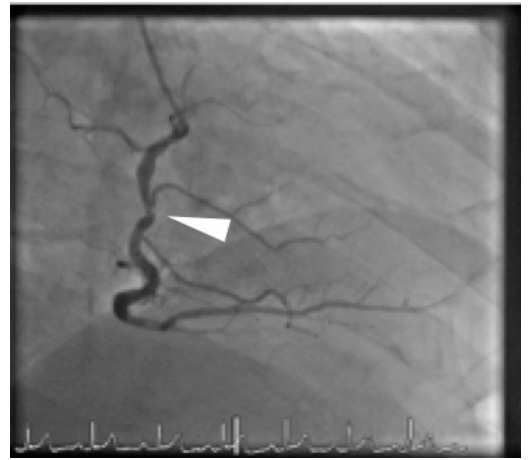

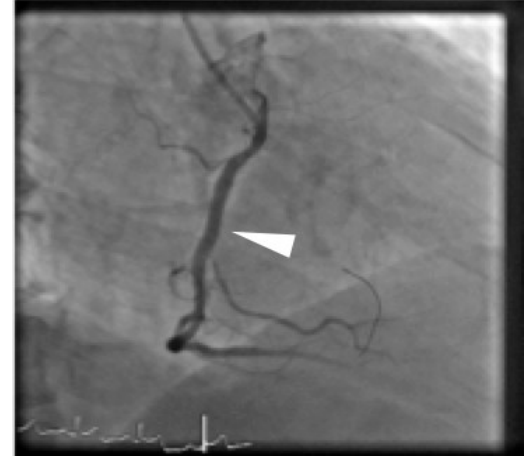

B

Figure 3. A. Mid RCA lesion pre-stenting with acute marginal branch (AMB). B. Mid RCA post-stenting with loss of acute marginal branch (AMB)

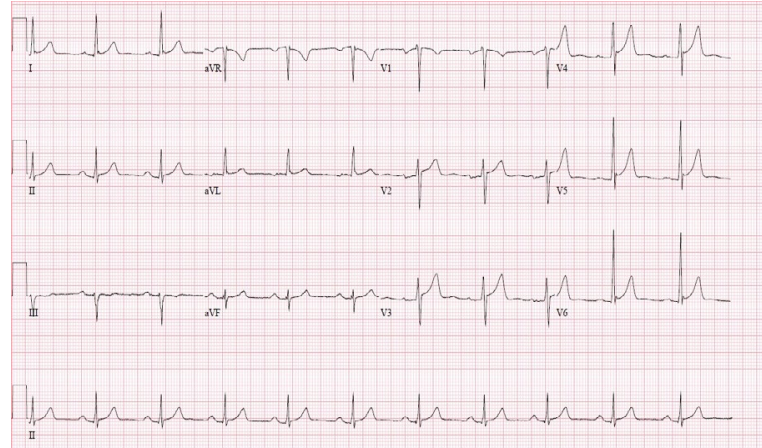

A

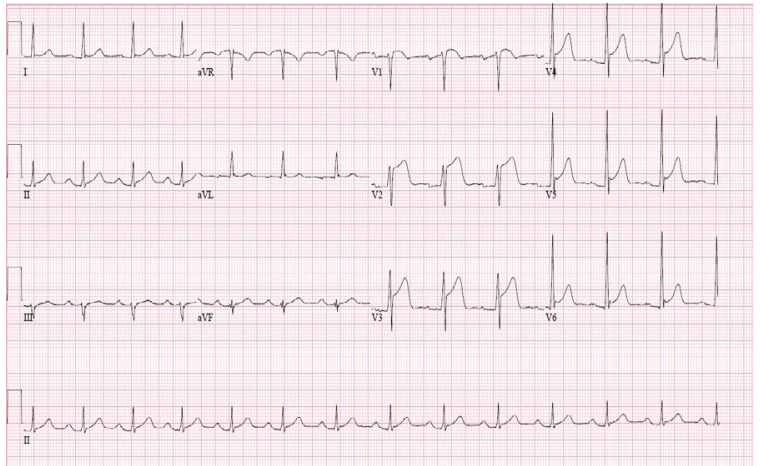

B

Figure 4. A. Baseline 12L ECG. B. 12L ECG showing 2mm ST-elevation of (V1-V3)

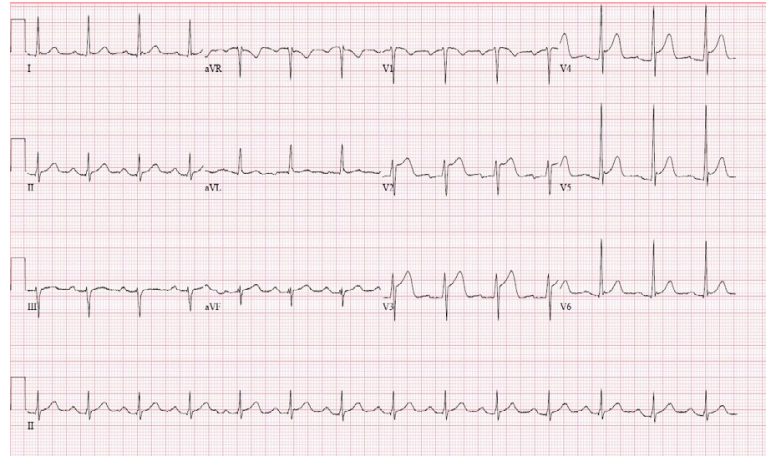

A. 1-hour post PCI

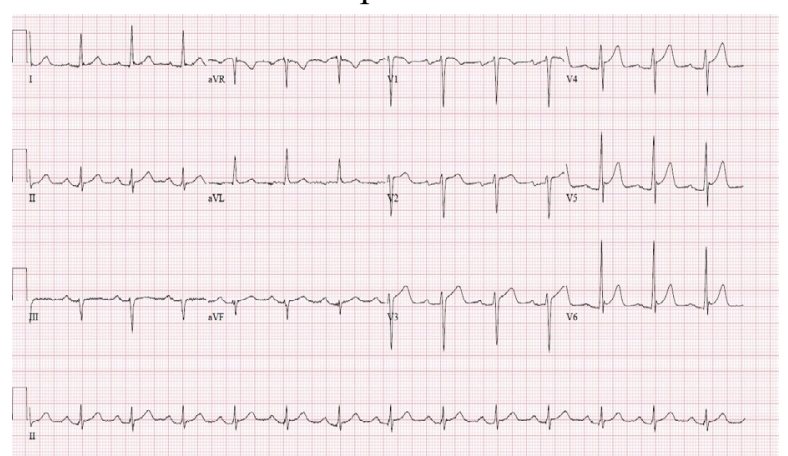

C. 9 hours post-PCI

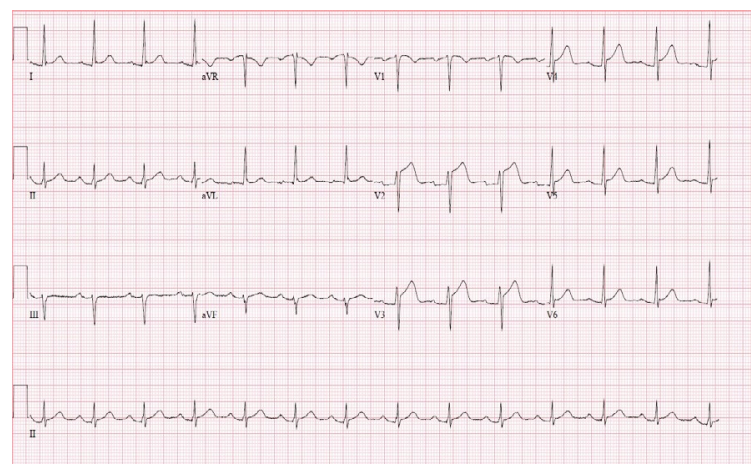

B. 2 hours post-PCI

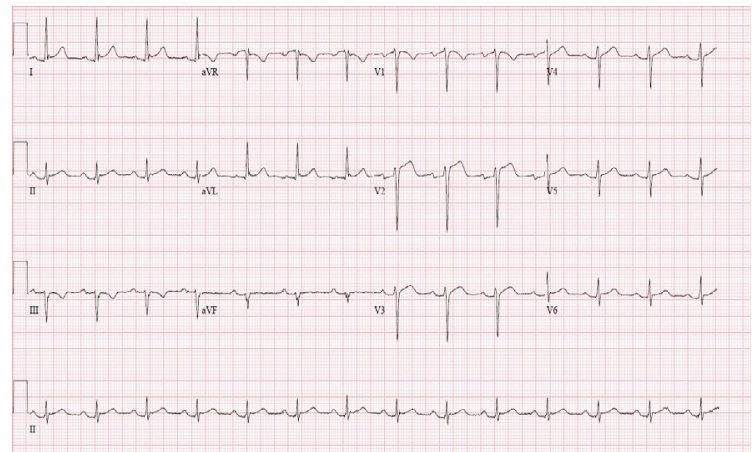

D. At discharge

Figure 5. (A-D) Serial ECG tracing post-PCI showing gradual resolution of anterior leads STE with no Q wave evolution and absence of concomitant inferior lead STE 
Tan KA (2021) Acute marginal branch occlusion presenting with anterior wall ST segment elevation during percutaneous coronary intervention of the mid right coronary artery

ventricle, the presence of minimal inferior wall current and the lack of posterior injury current permits the right ventricle injury current to predominate and manifest STE in leads V1-V4 [4]. Furthermore, the recording of

RV electrical potentials in the left precordial leads depends on the degree of clockwise rotation of the heart in the horizontal plane and on body geometry [5]. Isolated RV infarction (IRVI) is rare and occurs in less than $3 \%$ of all patients with myocardial infarction [6]. It may occur by i) proximal occlusion of a dominant right coronary artery (RCA), ii) occlusion of a nondominant RCA [7] and iii) isolated occlusion of an acute marginal branch (AMB) artery after percutaneous coronary intervention as demonstrated in our case.

IRVI should be suspected with the following ECG patterns: [7] (1) dome-like and decreasing ST segment elevation from leads V1 to V3; (2) rapid ST segment normalization and no Q- wave evolution from leads V1 to V3, with or without modest ST segment elevation in lead III (but not aVF) and no resultant Q wave; (3) ST segment elevation in right-sided leads; (4) absence of ST segment depression in aVL; (5) absence of concomitant ST segment elevation in inferior leads (II, III, aVF).

\section{Isolated anterior lead STE: IRVI versus anterior wall MI}

Anterior acute myocardial infarction (AMI) typically presents with anterior STE with reciprocal changes in the inferior leads [8]. This pattern of concomitant inferior ST segment depression is especially noted among AMI's involving proximal left anterior descending (LAD) coronary artery occlusion $[9,10]$ It is however important to note that absence of reciprocal changes in the inferior leads does not automatically rule out anterior wall AMI, as mid to distal LAD occlusion often still present with anterior STE with no reciprocal changes [9-11]. In addition, anterior wall AMI due to proximal LAD occlusion often presents with not only inferior ST segment depression but also STE in lead aVR or lateral limb leads, or both $[9,10]$. For patients with typical symptoms and ECG tracing of anterior lead STE, IRVI should be considered as a differential diagnosis [7,3]. Some authors have suggested a step wise approach in dealing with this finding [7,3]. First is to examine the presence of an incremental or decremental STE pattern from leads V1- V5. A decremental STE pattern favors IRVI [7]. Other investigators have considered the location of lead V1 being directly on top of RV, they have reported that STE primarily in V1 suggests IRVI [12]. Next is to look at the presence of ST depression (STD) in lead aVL. STD in aVL is a sign of reciprocal change in the presence of inferior wall injury, hence its absence points more towards the probability of IRVI [7]. Lastly, the presence of V3R and V4R STE makes the diagnosis IRVI highly probable [7]. No right sided precordial lead tracing was obtained in our case because of the information provided by coronary angiography while the anterior STE took place. Our patient however demonstrated a decremental STE pattern in V2-V4 and the absence of aVL STD. Serial ECG monitoring also did not show STE evolution into
Q waves in both the precordial and limb leads (Figure 5 A - D). These findings are consistent with the above mentioned patterns for IRVI.

\section{Conclusion}

Our case highlighted the importance of recognizing the possibility of IRVI as part of the differential diagnosis when presented with an ECG pattern suggestive of an anterior wall myocardial infarction. This is a rare case demonstrating isolated anterior lead STE mimicking anterior wall MI after loss of a mid RCA AMB during PCI. Consideration of IRVI prevented repeat left coronary circulation angiography and thus spared the patient from additional contrast and radiation. In the acute setting of primary PCI, keeping IRVI in mind along with careful angiographic review and ECG guidance may help correctly identify the culprit vessel when angiography to both left and right coronary circulations are equivocal and when lesions are of equal severity.

\section{References}

1. Klein HO, Tordjman T, Ninio R, Sareli P, Oren V, et al (1983) The early recognition of right ventricular infarction. Diagnostic accuracy of the electrocardiographic V4R lead. Circulation 67: 558-565. [Crossref]

2. Yano S, Mikuriya Y, Nasu M (1993) Influence of right ventricular ischemia on precordial ST depression during right coronary artery occlusion. Jpn Circ J 57: 803808. [Crossref]

3. Geft IL, Shah PK, Rodriges L, Hulse S, Maddahi J, et al. (1984) ST elevations in leads V1 to V6 may be caused by right coronary artery occlusion and acute right ventricular infarction. Am J Cardiol 53: 991-996. [Crossref]

4. Erol MK, Kocaturk H, Gurlertop Y (2005) Precordial ST segment elevation suggestive of anterior infarction caused by right coronary artery occlusion. Heart 91:1134. [Crossref]

5. Lew AS, Maddhai J, Shah PH, Weiss AT, Peter T, et al. (1985) Factors that determine the direction and magniture of precordial ST-segment deviations during inferior wall acute myocardial infarction. Am J Cardiol 55: 883-888. [Crossref]

6. Kinch JW, Ryan TJ (1994) Right ventricular infarction. N Engl J Med 330: 1211-1217 [Crossref]

7. Iannetta P, Puddu PE, Missiroli B, Morabito G, Grillo P, et al. (2013) Pathophysiology and ECG patterns of isolated right ventricular infarction with nondominant right coronary artery. J Cardiovasc Med 14: 740-744. [Crossref]

8. Harnett DT, LaHaye SA, Wilkinson JS (2016) Isolated right ventricular myocardia infarction: a sheep in wolf's clothing. JAMA Intern Med $176: 1207-1210$. [Crossref]

9. Engelen DJ, Gorgels AP, Cheriex EC, De Muinck ED, Ophuis AJ, et al. (1999) Value of the electrocardiogram in localizing the occlusion site in the left anterior descending coronary artery in acute anterior myocardial infarction. J Am Coll Cardiol 34: 389-395. [Crossref]

10. Tamura A, Kataoka H, Mikuriya Y, Nasu M (1995) Inferior ST segment depression as a useful marker for identifying proximal left anterior descending artery occlusion during acute anterior myocardial infarction. Eur Heart J 16: 1795-1799. [Crossref]

11. Walsh BM, Smith SW (2016) Differences in Electrocardiographic Findings Between Acute Isolated Right Ventricular Myocardial Infarction and Acute Anterior Myocardial Infarction. JAMA Intern Med 176: 1875-1876. [Crossref]

12. Kosuge M, Kimura K (2016) Differences in electrocardiographic findings between acute isolated right ventricular myocardial infarction and acute anterior myocardial infarction. JAMA Intern Med 176: 1875.

Copyright: (C2021 Tan KA. This is an open-access article distributed under the terms of the Creative Commons Attribution License, which permits unrestricted use, distribution, and reproduction in any medium, provided the original author and source are credited. 